\title{
Impact of Electron Collision Mixing on the Delay Times of an Electron Beam Excited Atomic Xenon Laser
}

\author{
PETER J. PETERS, YUN FU LAN, MIEKO OHWA, AND MARK J. KUSHNER, SENIOR MEMBER, IEEE
}

\begin{abstract}
The atomic xenon $(5 d \rightarrow 6 p)$ infrared laser has been experimentally and theoretically investigated using a short pulse ( $30 \mathrm{~ns}$ ) high power (1-10 MW/ $\mathrm{cm}^{3}$ ) coaxial electron beam excitation source. In most cases, laser oscillation is not observed during the $e$-beam current pulse. Laser pulses of 100 's of ns duration are subsequently obtained, however, with oscillation beginning 60-800 ns after the current pulse terminates. Results from a computer model for the xenon laser reproduce the experimental values, and show that oscillation begins when the fractional electron density decays below a critical value of $\approx 0.2-0.8 \times 10^{6}$. These results lend credance to the proposal that electron collision mixing of the laser levels limits the maximum value of specific power deposition which may be used to efficiently excite the atomic xenon laser on a quasi-CW basis.
\end{abstract}

\section{INTRODUCTION}

$\mathrm{T}$ $\mathrm{HE}$ atomic xenon infrared laser $(1.73-3.65 \mu \mathrm{m})$ is a versatile system which has yielded intrinsic power efficiencies in excess of $5 \%$. The xenon laser has been operated at pressures of $0.5-16$ atm in mixtures consisting of $\leq 0.5-2 \%$ of xenon in rare gas buffers ( $\mathrm{Ar}, \mathrm{He}, \mathrm{Kr})$. Electric discharge [1], [2], electron beam ( $e$ beam) [3], [4], $e$-beam sustained discharge [5], [6], and fission fragment [7] excitation at power depositions of $<10$ 's W $/ \mathrm{cm}^{3}$ - atm to $>\mathrm{MW}$ 's $/ \mathrm{cm}^{3} \cdot$ atm and pulse lengths of 10 's ns to $5 \mathrm{~ms}$ have been investigated. The most widely studied mixture has been $\mathrm{Ar}-\mathrm{Xe}$, whose dominant transitions are at $1.73 \mu \mathrm{m}\left(5 d[3 / 2]_{1} \rightarrow 6 p[5 / 2]_{2}\right)$ and $2.63 \mu \mathrm{m}$ $\left(5 d[5 / 2]_{2} \rightarrow 6 p[5 / 2]_{2}\right)$ with the $1.73 \mu \mathrm{m}$ line generally carrying the majority of the power (see Fig. 1). Lesser contributions are obtained from the transitions at $2.03 \mu \mathrm{m}$ $\left(5 d[3 / 2]_{1} \rightarrow 6 p[3 / 2]_{1}\right)$ and $2.65 \mu \mathrm{m}\left(5 d[3 / 2]_{1} \rightarrow\right.$ $\left.6 p[1 / 2]_{0}\right)$. In general, pumping significantly above threshold yields the majority of laser power at $1.73 \mu \mathrm{m}$.

Manuscript received May 2, 1990. The work of P. J. Peters and Y. F. Lan is part of the research program of the "Stichting voor Fundamenteel Onderzoek der Materie (FOM)," which is supported by the "Nederlandse organisatie voor Wetenschappelijk Onderzoek (NWO)". The work of M. Ohwa and M. J. Kushner was supported by Sandia National Laboratory.

P. J. Peters is with the Department of Applied Physics, University of Twente, 7500 AE Enshede, The Netherlands.

Y. F. Lan was with the Department of Applied Physics, University of Twente, $7500 \mathrm{AE}$ Enshede, The Netherlands. He is now with the Institute of Electronics, Academia Sinica, Beijing, People's Republic of China.

M. Ohwa was with the Department of Electrical and Computer Engineering, University of Illinois, Champaign, IL 61820. She is now with the Department of Electrical Engineering, Keio University, Yokahama, Japan.

M. J. Kushner is with the Department of Electrical and Computer Engineering, University of Illinois, Urbana, IL 61801.

IEEE Log Number 9030190.
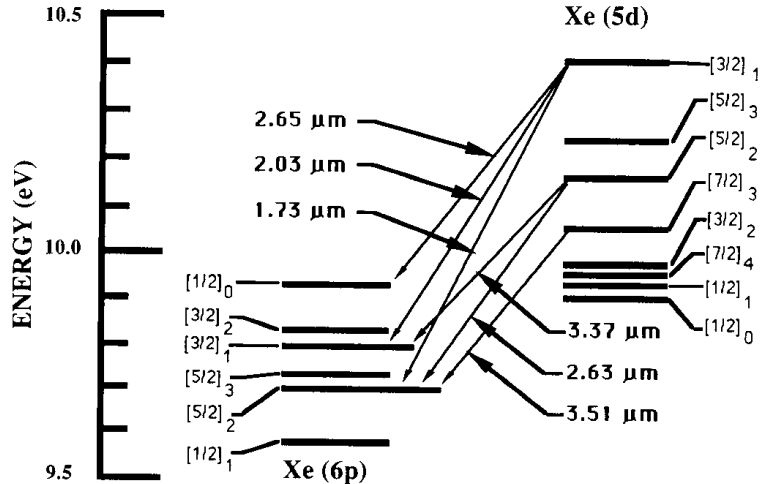

Fig. 1. Energy levels of Xe showing the laser transitions. In Ar-Xe mixtures, the $1.73 \mu \mathrm{m}$ transition dominates.

When pumping near threshold the $2.03 \mu \mathrm{m}$ transition is more important due to its higher oscillator strength relative to the $1.73 \mu \mathrm{m}$ transition. In high pressure $(>0.5$ atm ) Ar-Xe mixtures, the upper laser level is most likely excited by a collisional-radiative cascade following dissociative recombination of $\mathrm{ArXe}^{+}$. Recombination directly populates high lying excited states, denoted $\mathrm{Xe}^{* *}$, which cascade to the $5 d$ manifold dominantly by heavy particle collisions [3]. The $6 p$ manifold is also dominantly quenched by heavy particle collisions. In fact, the rapid quenching of the common lower laser level of the 1.73 and $2.63 \mu \mathrm{m}$ transitions $\left(6 p[5 / 2]_{2}\right)$ in Ar-Xe mixtures allows both to simultaneously oscillate with little competition.

The fact that the laser transitions have large oscillator strengths and their energy difference is less than $1 \mathrm{eV}$ suggests that electron collisional mixing (ECM) of the laser levels may be important. Experimental and theoretical evidence for the importance of ECM has appeared in the literature however definitive experiments have not previously been performed. For example, it has been observed that the saturation intensity of the $1.73 \mu \mathrm{m}$ transition increases proportional to $P^{0.6}$ ( $P$ is the power deposition) in $e$-beam and fission fragment excited systems. This behavior suggests that ECM dominates the lifetime of the inversion since in recombination dominated plasmas $n_{e}-$ $P^{0.5}$. The laser has also been observed to terminate prior to the end of excitation in long pulse ( $1 \mathrm{~ms}$ ), low power 
deposition ( $\left.\leq 1 \mathrm{~kW} / \mathrm{cm}^{3}\right)$ systems [4]. This effect has been attributed to the increase in electron density, and hence ECM, experienced at high energy loading. Results from a model for the xenon laser predict that the optimum quasi-CW pump rate for $\mathrm{Ar}-\mathrm{Xe}$ mixtures is that value resulting in a fractional ionization of $\approx 2-3 \cdot 10^{-6}$. At pump rates resulting in a fractional ionization $>(0.8-1.0) \cdot$ $10^{-5}$ ECM quenches oscillation [8].

In this paper, experimental and theoretical results are presented which support the proposition that ECM limits the maximum fractional ionization, and hence power deposition, with which the atomic xenon laser may be operated on a quasi-CW basis. The experimental results are measurements of laser power from an $\mathrm{Ar}-\mathrm{Xe}=99.7: 0.3$ mixture excited by a short pulse ( $30 \mathrm{~ns}$ ) high power (1$10 \mathrm{MW} / \mathrm{cm}^{3}$ ) coaxial electron beam. The theoretical results are from a model for the xenon laser which simulates the $e$-beam excitation of $\mathrm{Ar}-\mathrm{Xe}$ mixtures and subsequent laser oscillation on five transitions between the $5 d$ and $6 p$ manifolds. Briefly, we found that laser oscillation is not observed during the $e$-beam current pulse but does subsequently occur $60-800$ ns after the current pulse during the afterglow. Results from the model show that laser oscillation begins after the electron density decays by recombination to a fractional ionization $\leq 1 \cdot 10^{-5}$. Previous to this time, electron collision mixing of the laser levels prevents oscillation. As a consequence there is a delay between the onset of the pumping pulse and the start of the laser output pulse. This delay time has been measured and the results will be described in this paper.

In Section II, the experimental apparatus and procedures will be described followed by a brief overview of the model in Section III. Experimental and theoretical results are compared in Section IV where the dominant kinetic processes are discussed. Our concluding remarks are in Section V.

\section{DESCRIPTION OF THE EXPERIMENT}

All experiments described in this paper were performed with a small tabletop coaxial $e$-beam apparatus. The system is able to homogeneously excite a high pressure gas using a coaxial beam of high energy electrons at a high current density. A cross-sectional view of the system is shown in Fig. 2. The electrons are produced inside an evacuated coaxial diode structure. The anode of this diode is a thin titanium tube with a wall thickness of either $\mathbf{5 0}$ or $25 \mu \mathrm{m}$. The inner diameter of the tube is $10 \mathrm{~mm}$. The diameter of the cylindrical volume from which laser power is extracted, however, is restricted to $9.2 \mathrm{~mm}$ due to the inner diameter of the ends of the tube. These solid end pieces are laser welded to the thin titanium tube in order to be able to mount the tube and make an appropriate pressure seal. In order to withstand the high gas pressure inside the tube we reinforced the tube by using a tungsten wire of $0.25 \mathrm{~mm}$ diameter. The wire was wound as a spiral tightly over the tube surface with a pitch of $5 \mathrm{~mm}$ and fixed onto the two solid tube ends. With this reinforce-

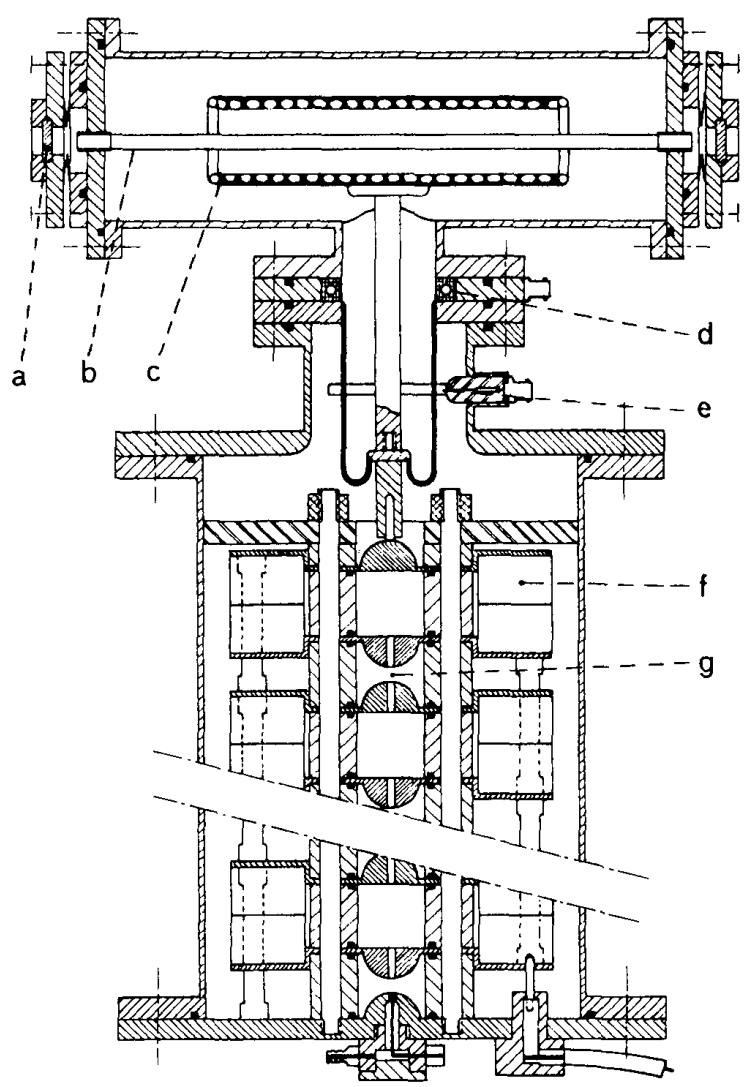

Fig. 2. Cross-sectional view of the coaxial $e$-beam device (a: mirror mount, b: titanium anode tube, c: cathode, d: current sensor, e: voltage sensor, f: doorknob capacitor, g: spark-gap).

ment we were able to work without failure at total gas pressures as high as 18 bar for several hundreds of shots.

The cathode consists of a $20 \mathrm{~cm}$ long metal cylinder having an inner diameter of $5 \mathrm{~cm}$. Two or three carbon felt strips with lengths of $20 \mathrm{~cm}$ each were mounted inside this cylinder which provided a good match between the Marx generator and the diode. The cathode was fed by a ten stage low inductance Marx generator enclosed in a cylindrical stainless steel vessel having a diameter of 25 $\mathrm{cm}$ and a height of $1 \mathrm{~m}$. The vessel was pressurized with 4 bar $\mathrm{SF}_{6}$ to prevent internal flashover. Each stage of the Marx generator is comprised of eight pairs of cylindrical $\mathrm{BaTiO}_{3}$ capacitors of $1.8 \mathrm{nF}$ each yielding a total capacitance of $7.2 \mathrm{nF}$. The maximum allowed load voltage is $60 \mathrm{kV} /$ stage. The coaxial and compact construction of the Marx generator provides a low inductance and a fast rise time for the $e$-beam pulse. The total stored energy in the Marx generator is about $100 \mathrm{~J}$ at the maximum load voltage of $60 \mathrm{kV}$. The voltage is monitored by a differentiating voltage sensor while the current is measured by a self-integrating Rogowski coil. Typical plots of the diode current and voltage at a load voltage on the Marx generator of $45 \mathrm{kV}$ are shown in Fig. 3.

The optical resonator has length of $40 \mathrm{~cm}$ and consists 

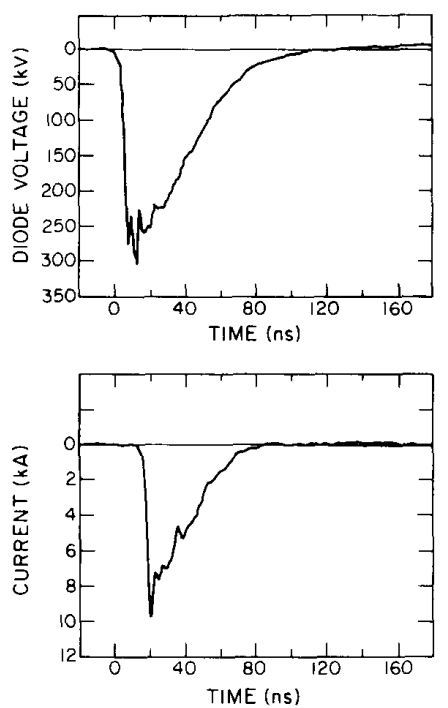

Fig. 3. Diode voltage and current for a Marx load voltage of $45 \mathrm{kV}$.

of a gold coated total reflecting mirror with a radius of curvature of $2 \mathrm{~m}$ on one side and a plan parallel uncoated $\mathrm{ZnSe}$ flat on the other side. The laser output energy was measured by a Laser Precision (RJP735-RJ7200) detector. The spectral composition of the laser light was studied using a Hilger $8 \mathrm{~W}$ monochromator with a focal length of $30 \mathrm{~cm}$ equipped with a $1501 / \mathrm{mm}$ grating blazed at 4 $\mu \mathrm{m}$. The dispersed signal was detected by an uncooled InSb ORP 10 detector.

\section{Description of the Model}

The computer model we used to analyze the experimental results consists of an accounting of the electron and heavy particle kinetics in the xenon laser in $\mathrm{Ar}-\mathrm{Xe}$ mixtures using $e$-beam excitation. It is functionally equivalent to the model described in [8] and therefore will be only briefly described here. A Monte Carlo simulation is used to generate electron impact rate coefficients for ionization and excitation by the $e$ beam for a given gas mixture. Those rate coefficents are used as input to a kinetics model which calculates the time dependent density of electrons, ions and excited states. Twenty levels of the xenon atom are in the model including all levels in the $6 p$ and $5 d$ manifolds. A listing of the important rate coefficients for electron impact and heavy particle collisions for these levels is given in [8]. Laser oscillation on five infrared transitions between the $5 d$ and $6 p$ manifolds are also included in the model.

Electron collision mixing between all levels of the $5 d$ and $6 p$ manifolds was included in the model. A single rate coefficient was used for all exothermic transitions and detailed balance was used for the reverse process. The rate coefficients for electron collision mixing between levels $i$ and $j$ of the $5 d$ and $6 p$ manifold are

$$
k_{i j}=k_{0} \quad \epsilon_{i}>\epsilon_{j}
$$

and for the reverse processes

$$
k_{j i}=\frac{g_{i}}{g_{j}} \cdot k_{0} \exp \left[-\frac{\epsilon_{i}-\epsilon_{j}}{k T_{e}}\right]
$$

where $\epsilon_{i}$ is the energy of the level $i, g_{i}$ is its degeneracy and $T_{e}$ is the electron temperature. The electron temperature is $1-2 \mathrm{eV}$ during the current pulse and decays to tenths of an $\mathrm{eV}$ during the afterglow. We used $k_{0}=2$. $10^{-7} \mathrm{~cm}^{3} \mathrm{~s}^{-1}$, a value derived in [8].

\section{Time Delay for Oscillation}

\section{A. Experimental Results}

As discussed below power deposition is an important parameter in the analysis of our experiment. In order to estimate this parameter we measured the energy deposition in the laser gas mixture using the pressure jump technique. The excitation volume was $51 \mathrm{~cm}^{3}$. We used a LX $1702 \mathrm{DZ}$ differential pressure transducer from National Semiconductor (now: Sen Sym). Typical output signals are shown in Fig. 4. The lower curve is the transducer signal from a 9 bar He fill while the upper trace was obtained from 9 bar $\mathrm{Ar}-\mathrm{Xe}=99.5: 0.5$ mixture. The Marx load voltage for both was $60 \mathrm{kV}$ and a $25 \mu \mathrm{m}$ Titanium tube was used as the anode.

It is clear from this figure that there can be a balance between the heat deposited in the anode tube and in the gas. If the heat deposition in the gas is relatively low, additional gas heating occurs from the heated anode foil. This occurs with the He gas fill as can be seen in the lower trace of Fig. 4. On the other hand a gas mixture with a high stopping power like Ar-Xe $=99.5: 0.5$ can be cooled by the relatively cool anode tube wall as shown in the upper trace of Fig. 4. The energy deposition was calculated for various gasses at different pressures and at different Marx load voltages by using the transducer signals at $t=0$. The power deposition was calculated by assuming that the pump pulse is rectangular with a pulse width of $30 \mathrm{~ns}$. The measured power deposition is plotted as a function of the total Ar pressure in Fig. 5 for various Marx load voltages for a titanium anode tube of $50 \mu \mathrm{m}$ thickness. In order to quench $\mathrm{Ar}_{2}^{*}$ emission $0.5 \%$ of $\mathrm{Xe}$ (or $1.5 \% \mathrm{HCl}$ ) was added to Ar during the pressure jump measurements.

Next the laser output energy was measured for various $\mathrm{Xe}$ concentrations at different total gas pressures and at different power deposition rates. The highest output energy was obtained with a $25 \mu \mathrm{m}$ titanium tube and gas mixtures containing $0.3 \%-0.5 \% \mathrm{Xe}$ in Ar. The optimum total output energy occured at relatively low power deposition rates as can be seen in Fig. 6. The delay times between the start of the current pulse and start of oscillation as a function of the power deposition have been 


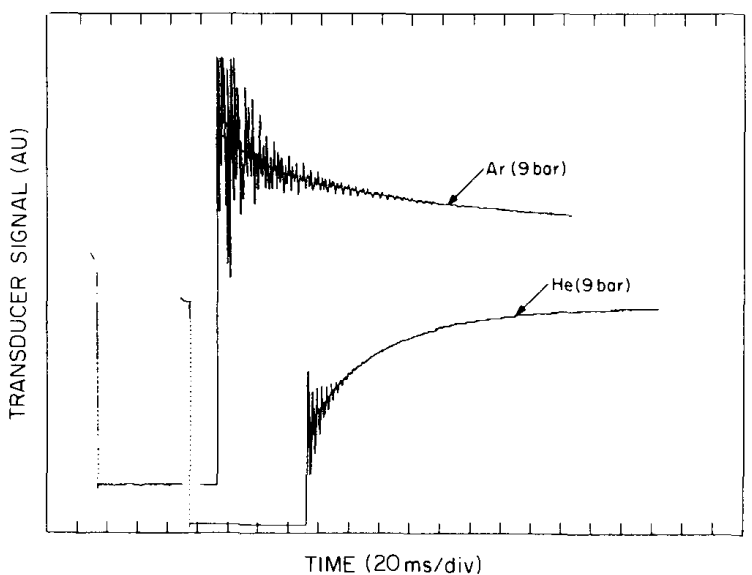

Fig. 4. Response of the pressure transducer upon $e$-beam firing at ou $\mathrm{kV}$ load voltage. The gas fills are 9 bar $\mathrm{Ar}-\mathrm{Xe}: 99.5: 0.5$ (upper trace) and 9 bar He (lower trace).

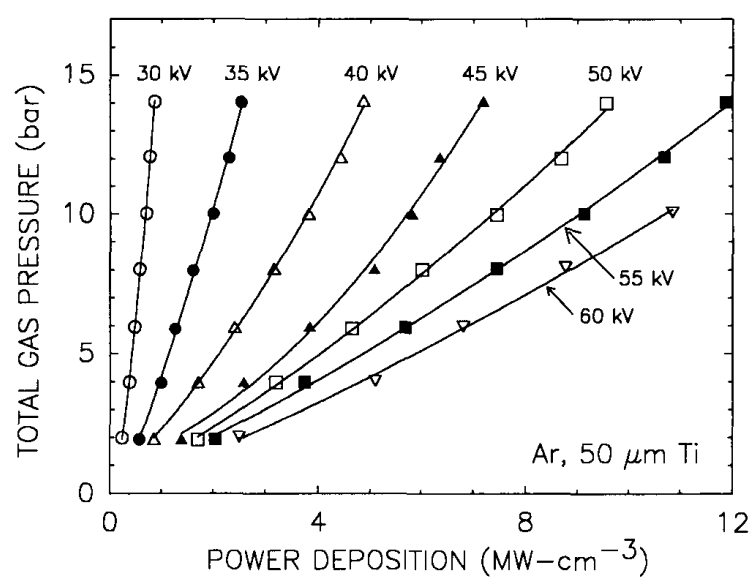

Fig. 5. Power deposition in Ar-Xe: $99.5: 0.5$ as a function of the total gas pressure at different Marx load voltages.

measured for the four strongest laser lines. The gas mixture was $0.3 \% \mathrm{Xe}$ in $\mathrm{Ar}$ and the wall thickness of the anode tube was $50 \mu \mathrm{m}$. The results are plotted for the $\lambda=1.73 \mu \mathrm{m}$ laser line in Fig. 7. The results from the model are also plotted as solid lines in this figure. The measured delay times increase with increasing power deposition and the agreement with the model is fairly good. Experimental results obtained for $\lambda=2.63 \mu \mathrm{m}$ are almost identical to those for $1.73 \mu \mathrm{m}$ laser line and are also in agreement with the model. A discussion of these trends using results from the model appears in Section IV-B. The time delays to oscillation for the $2.65 \mu \mathrm{m}$ laser line showed two distinct features. There appears to be a direct pumped channel for this line which is suggested by the fact that there is a constant time delay of about $40 \mathrm{~ns}$ for all pressures and pumping rates. This first peak in laser

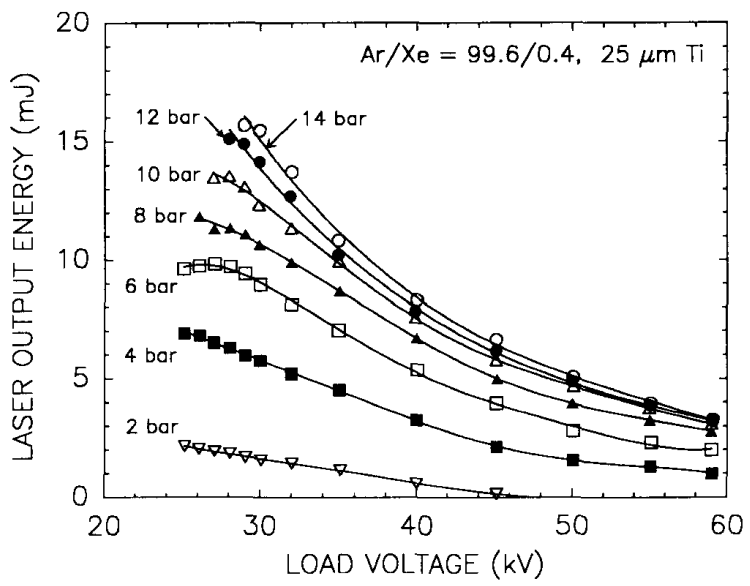

Fig. 6. Output energy from a laser volume of $15.7 \mathrm{~cm}^{3}$ as a function of the Marx load voltage at different total gas pressure (Ar-Xe $=99.6: 0.4$ $25 \mu \mathrm{m}$ titanium anode).

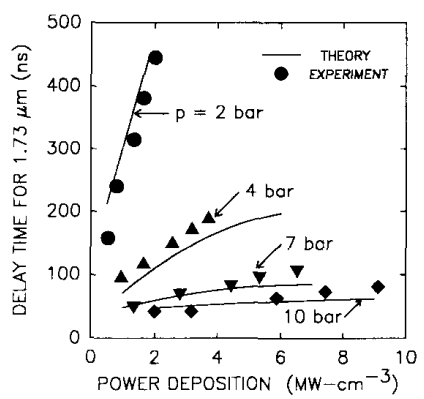

Fig. 7. The time delay to oscillation at $1.73 \mu \mathrm{m}$ after the start of the current pulse in an $\mathrm{Ar}-\mathrm{Xe}=99.7: 0.3$ mixture as a function of average power deposition during a $30 \mathrm{~ns}$ excitation pulse. The points are from the experiment; the solid lines are results from the model.

power is sometimes followed by a second peak having nearly the same width of roughly $50-100 \mathrm{~ns}$. The delay times of the following main peaks show more or less the same behavior as the 1.73 and $2.63 \mu \mathrm{m}$ lines. A totally different behavior was found for the $\lambda=3.37 \mu \mathrm{m}$ line, as can be seen in Fig. 8. Here the delay times decrease with increasing power deposition rates. It appeared that for all gas pressures a much higher energy deposition was required to obtain the optimum output energy for the 3.37 $\mu \mathrm{m}$ line compared to the $1.73 \mu \mathrm{m}$ line.

The output energy of the individual lines could not be measured on an absolute scale due to the wavelength dependent character of some optical components, such as the grating and the detector. However a rough estimate could be made for the change of the output energy of the different lines under various pumping conditions by calculating the time integral of the detector signals.

We found that the ratio of the optima of the integrated signals of the 1.73 to the $2.63 \mu \mathrm{m}$ line decreased going 


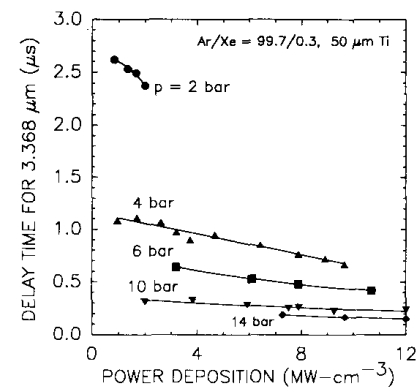

Fig. 8. Time delay to oscillation after the start of the current pulse of the $3.37 \mu \mathrm{m}$ line as a function of the average power deposition.

from 2 to 14 bar. So at a higher gas pressure the contribution of the $2.63 \mu \mathrm{m}$ line to the total optical output energy increased at the expense of the $1.73 \mu \mathrm{m}$ contribution. Comparing the energies of the 2.63 and $2.65 \mu \mathrm{m}$ lines, their contributions to the total output energy were found to be roughly the same over the whole pressure range. The ratio of the integrated signals of the 1.73 to the $3.37 \mu \mathrm{m}$ lines was almost constant at a pressure above 7 bar. Going to lower pressures the ratio increased steeply mainly due to the very strong decrease of $3.37 \mu \mathrm{m}$ signal.

\section{B. Model Results and Analysis}

The experiments were simulated using the model described in Section III. The delay times to oscillation obtained with the model for the $1.73 \mu \mathrm{m}$ transition are shown as the solid lines in Fig. 7. The experimental results are fairly well reproduced, and may be explained by referring to Fig. 9(a). In this figure we show the computed electron density as a function of time for a pressure of 7 bar for different pump powers. The time at which oscillation begins in each case is shown by the circles in the inset. In Fig. 9(b) the fractional ionization at the time at which the laser turns on is shown as a function of pump rate and gas pressure. At each pressure the laser does not begin to oscillate until the fractional ionization falls below a specific value which depends upon pressure. The range is $2-6$. $10^{-6} \mathrm{~cm}^{-3}$. The interpretation of these results is that electron collision mixing of the laser levels prevents oscillation until the electron density falls below a specific fractional ionization. The effect scales with fractional ionization instead of absolute electron density. This scaling results from the fact that the inversion is formed by pumping into the $5 d$ manifold by a cascade from higher lying levels by heavy particle collisions. The $6 p$ manifold is also dominantly cleared by heavy particle collisions. Therefore the detrimental effects of mixing of the levels by electron collisions are offset by heavy particle induced pumping of the upper laser level and clearing of the lower laser level. The maximum tolerable fractional ionization increases with increasing gas pressure for these reasons. The impact of ECM on the delay time can be shown by

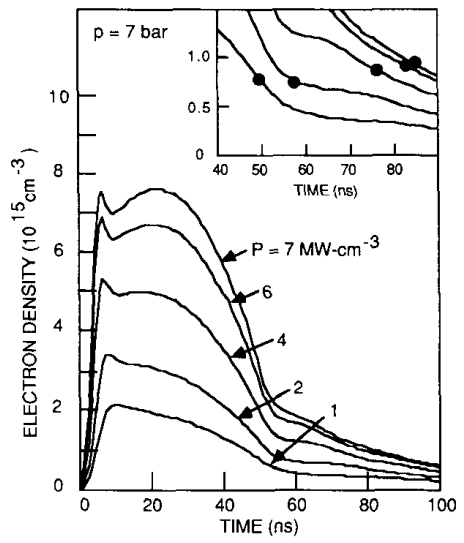

(a)

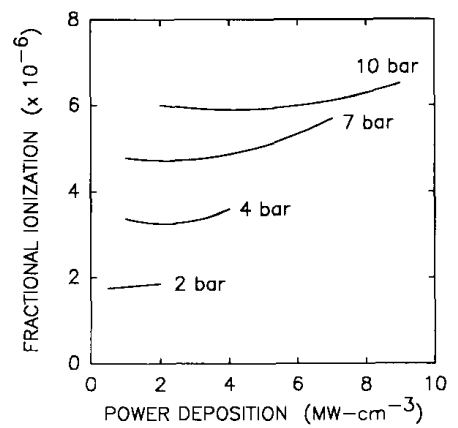

(b)

Fig. 9. (a) Computed electron density for different average power depositions as a function of time at a pressure of 7 bar. The circles in the inset shown the electron density at which oscillation begins. (b) Fractional ionization at the time at which oscillation begins as a function of average power deposition at different pressures. In each case, the fractional ionization at threshold is nearly constant.

excluding that process from the model and repeating the simulations discussed above. For example, at a gas pressure of 7 bar the delay time in the absence of ECM is virtually constant as a function of power deposition (12$14 \mathrm{~ns}$ ) up to $6 \mathrm{MW} \mathrm{cm}^{-3}$. The delay with ECM is $40-80$ ns.

The delay time to oscillation increases with increasing power deposition at a given pressure because the maximum electron density during the current pulse scales roughly as $P^{0.5}$. Therefore a longer time is required for the electron density to decay below its critical value for a given pressure as the power deposition increases. The delay time also increases with decreasing gas pressure. This trend results from two causes. The first is that electron loss is dominantly by dissociative recombination of $\mathrm{ArXe}^{+}$and $\mathrm{Xe}_{2}^{+}$. Although the recombination event is itself a two-body process, the association reactions forming $\mathrm{ArXe}^{+}$and $\mathrm{Xe}_{2}^{+}$are three-body processes. Therefore the rate of formation of dimer ions, and hence recombination, decreases with decreasing gas pressure. Since the electron 
density must decrease below a critical value to reach threshold, this time delay increases with decreasing gas pressure.

The second cause is a consequence of the manner in which the $5 d$ manifold is populated. Recombination of $\mathrm{ArXe}^{+}$, populates highly excited states of $\mathrm{Xe}$, denoted $\mathrm{Xe}^{* *}$. Cascade of $\mathrm{Xe}^{* *}$ to the $5 d$ manifold progresses by radiation and heavy particle quenching. Therefore the higher the gas pressure, the higher the rate of population of the $5 d$ manifold by collisional cascade. To investigate the contribution of the cascade time to the total delay we parameterized the model by using $[e]=\left[\mathrm{ArXe}^{+}\right]=10^{15}$ $\mathrm{cm}^{-3}$ as an initial condition. No further power deposition or lasing was allowed. The density of $\operatorname{Xe}\left(5 d[3 / 2]_{1}\right)$ is plotted in Fig. 10(a) as a function of time for gas pressures of $2-10$ bar. The time at which the density is a maximum is plotted in Fig. 10(b). The cascade time is short, 5-15 ns and decreases with increasing gas pressure. As this time is short compared to the total delay time (10's $\mathrm{ns}$ ), the majority of the delay is therefore due to the time required for the electron density to decay below its critical value. The peak $\mathrm{Xe}\left(5 d[3 / 2]_{1}\right)$ density following the cascade has a weak maximum near 6 bar. It decreases towards lower pressure due to radiative decay of higher levels having branchings to levels other than the $5 d$ manifold.

The cascade time shown in Fig. 10(b) is important because it sets on upper limit on the duration with which one may pump at power levels resulting in electron densities exceeding the critical value without decreasing laser energy efficiency. For example, suppose that the current pulse is long compared to the cascade and recombination times, and the electron density is greater than the critical value $n_{c}$. Then deposited energy which is channeled to the $5 d$ manifold is therefore wasted due to ECM which prevents oscillation. If however, the current pulse is short compared to the recombination and cascade time, then energy flowing into the $5 d$ manifold is available for laser extraction without interference from ECM. An indication of how the efficiency of the laser may scale under these conditions is therefore the fraction of the deposited energy which resides in the excited states and ions of the gas $\beta$ at the time at which $n_{e}$ decreases below $n_{c}$. $\beta$ as calculated with the model is plotted in Fig. 11 as a function of time. $\beta$ decreases with time after the current pulse as excited states are quenched or radiate. The value of $\beta$ at the time at which laser threshold occurs is shown in Fig. 12. At the time the laser reaches threshold for a pump power of $1 \mathrm{MW} \mathrm{cm} \mathrm{cm}^{3} \beta=0.3$, a value which decreases to 0.08 at a pump power of $4 \mathrm{MW} \mathrm{cm} \mathrm{cm}^{-3}$. One would therefore expect laser efficiency to similarly scale over the same range; that is, a monotonic decrease in laser efficiency with increasing power deposition. This trend is observed experimentally as shown in Fig. 12.

Laser efficiency and $\beta$ scale quite similarly as a function of power, and hence energy deposition. Laser energy efficiency for our short excitation pulse conditions therefore

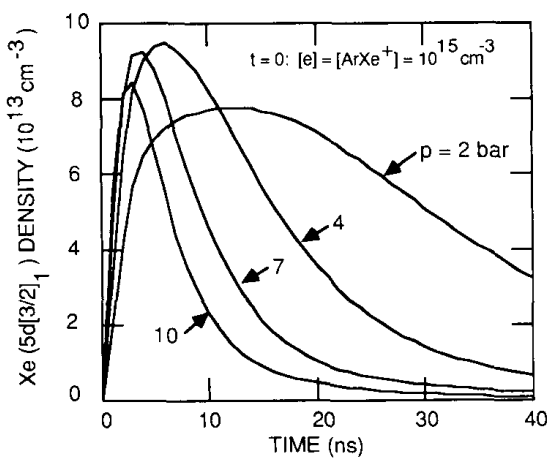

(a)

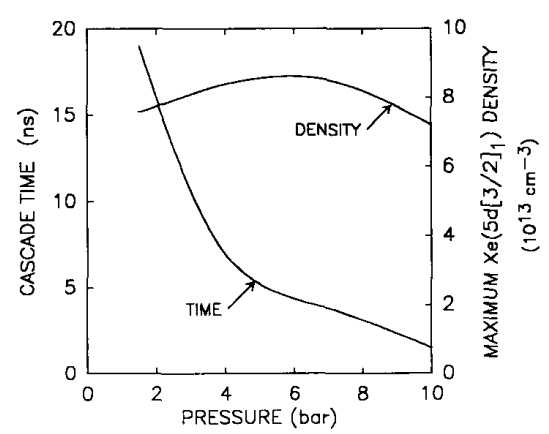

(b)

Fig. 10. Results of a computer experiment to investigate the collisional cascade following recombination to populating $\mathrm{Xe}\left(5 d[3 / 2]_{1}\right)$ populated states. Initial conditions are $[e]=\left[\mathrm{ArXe}^{+}\right]=10^{1.5} \mathrm{~cm}^{-3}$. (a) Density of $\mathrm{Xe}\left(5 d[3 / 2]_{1}\right)$, upper laser level for the $1.73 \mu \mathrm{m}$ transition, as a function of time, at different pressures. (b) Cascade time, defined as the time to the peak in the $\operatorname{Xe}\left(5 d[3 / 2]_{1}\right)$ density, as a function of gas pressure. The maximum excited state density is also shown, which has a weak maximum near $p=4$ bar.

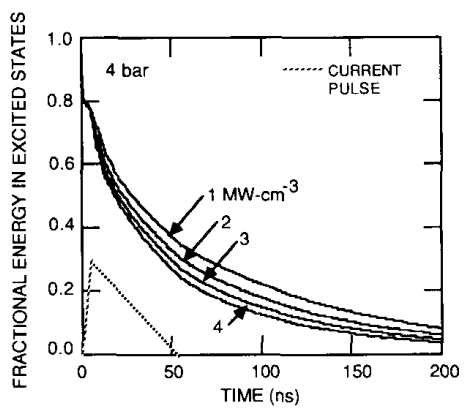

Fig. 11. Calculated fraction of deposited energy which resides in excited states $\beta$ for a pressure of 4 bar at different power depositions. The current pulse is shown as the dashed line.

is determined in large part by the fraction of deposited energy which can be stored in the excited state manifold at the time of laser threshold. 


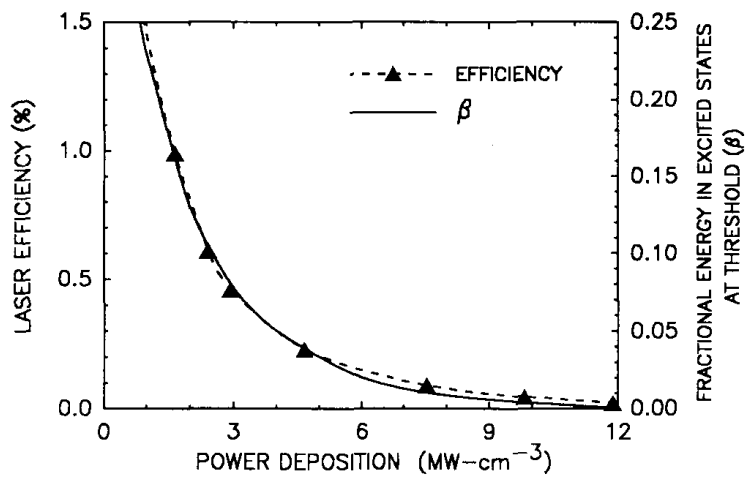

Fig. 12. Laser efficiency and the calculated fraction of energy which resides in excited states $\beta$ as a function of average power deposition $(p=4$ bar $\mathrm{Ar}-\mathrm{Xe}=99.7: 0.3)$

\section{Concluding Remarks}

An experimental and theoretical study of the atomic xenon laser using short pulse high power excitation has been performed to investigate excitation and quenching mechanisms of the $5 d$ manifold of xenon which serves as the upper laser level. The delay time before oscillation begins results from the necessity for the electron density to decay below a critical value $\left([e] / N \approx 2-8 \cdot 10^{-6}\right)$ above which electron collision mixing dominates. Our results are consistent with a set of reactions in which excitation of the upper laser level follows a heavy particle collisional cascade after recombination of $\mathrm{ArXe}^{+}$. Mixing of the laser levels is dominated by electron collisions, and clearing of the lower laser level is dominantly by heavy particle collisions.

\section{ACKNOWLEDGMENT}

The authors would like to acknowledge helpful conversations with Dr. E. Patterson, Dr. G. N. Hays, and A. J. Alford.

\section{REFERENCES}

[1] F. S. Collier, P. Labastie, M. Maillet, and M. Michon, "High-efficiency infrared xenon laser excited by a UV preionized discharge," IEEE J. Quantum Electron., vol. QE-19, pp. 1129-1133, 1983.

[2] J. E. Tucker, B. L. Wexler, B. J. Feldman, and T. McClelland, "High pressure infrared xenon laser with X-ray preionization," IEEE Photon. Techonol. Lett., vol. 1, pp. 193-195, 1989.
[3] P. J. M. Peters, Q. -C. Mei, and W. J. Witteman, "Pressure dependent optical delay time measurements in a coaxial electron beam pumped Ar/Xe laser," Appl. Phys. Lett., vol. 54, pp. 193-195, 1989.

[4] E. L. Patterson, G. E. Samlin, and P. J. Brannon, "Results of atomic Xe laser operation at high energy loadings," Sandia Rep., Sand89-0716; UC 414, 1989.

[5] S. A. Lawton, J. B. Richards, L. A. Newman, L. Specht, and T. A De Temple, "The high-pressure neutral infrared xenon laser," J. Appl. Phys., vol. 50, pp. 3888-3898, 1979.

[6] N. G. Basov, V. V. Baranov, A. Y. Chugunov, V. A. Danilychev, A Y. Dudin. I. V. Kholin, and N. N. Ustinovskii, "60 J quasi-stationary electroionization laser on Xe atomic metastables," IEEE J. Quantum Electron., vol. QE-21, pp. 1756-1760, 1985.

[7] W. J. Alford and G. N. Hays, "Measured laser parameters for reactorpumped $\mathrm{He} / \mathrm{Ar} / \mathrm{Xe}$ and $\mathrm{Ar} / \mathrm{Xe}$ lasers," J. Appl. Phys., vol. 65, pp. 3760-3766, 1989.

[8] M. Ohwa, T. J. Moratz, and M. J. Kushner, "Excitation mechanisms of the electron-beam-pumped atomic xenon $(5 d-6 p)$ laser in $\mathrm{Ar} / \mathrm{Xe}$ mixtures," J. Appl. Phys., vol. 66, pp. 5131-5145, 1989.

Peter J. Peters, for photograph and biography see p. 1572 of the September 1990 issue of this JoURNAL.

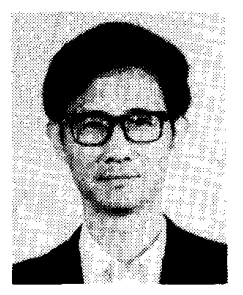

Yun Fu Lan received the equivalent M.A. degree from Tsinghua University, Beijing, China, in 1965

He then joined the Institute of Electronics Academia Sinica. Beijing. China, in 1965 and was engaged in the research of high-power microwave tubes at the Microwave Device Laboratory between 1966 and 1981. He became a visiting scholar and worked on electromagnetic field theory by computational simulation from April 1981 to January 1982 , at the University of Waterloo, Waterloo. Ont.. Canada. He was then at the Microwave Research Institute Polytechnic Institute of New York, New York City, NY, from January 1982 to July 1983 and worked on the development of high-power, highrepetition-rate $\mathrm{TEA} \mathrm{CO}_{2}$ lasers during the period 1985-1988, at the Laser Laboratory, Institute of Electronics, Academia Sinica. He became a research Associate Professor in 1986. Since May 1988, he has been involved in the research of electron-beam pumped atomic $\mathrm{Ar}-\mathrm{Xe}$ lasers at the Quantum Electronics Group, University of Twente, Enschede, The Netherlands $\mathrm{He}$ is a member of the Chinese Electronics Society.

Mieko Ohwa, for photograph and biography see p. 1646 of the September 1990 issue of this JoURNAL.

Mark J. Kushner (S'74-M'79-SM'89), for photograph and biography see p. 1554 of the September 1990 issue of this JournaL. 